\title{
Mucormicosis cutánea en un paciente inmunocomprometido
}

\author{
Marlis Täger F., Luis Zaror C. y Pilar Martínez D.
}

\section{Cutaneous mucormycosis in an immunocompromised patient}

Mucormycosis is an increasingly emerging life-threatening infection and is an important cause of morbidity and mortality in patients with leukemia. We present the case of a 4-year-old boy with an acute lymphoblastic leukemia complicated by severe and prolonged neutropenia during induction chemotherapy, who developed cutaneous mucormycosis of the elbow. Direct microscopy with $\mathrm{KOH}$ and the histopathologic observation of the skin revealed fungal hyphae without septations. The cultures were positive for Rhizopus microsporus var oligosporus. The patient was treated succefully with amphotericin B during 40 days, surgical debridement and dermoepidermic graft. Early recognition and prompt intervention with combined medical and surgical treatment may improve the outcome. The most common management strategy in survivors involves a combination of antifungal therapy with amphotericin B and surgical debridement.

Key words: Mucormycosis, cutaneous mucormycosis, fungal infections, immunocompromise, Rhizopus.

Palabras clave: Mucormicosis, mucormicosis cutánea, infecciones fúngicas, inmunocompromiso, Rhizopus.

\section{Introducción}

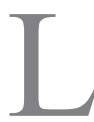

as micosis por hongos hialinos no tabicados se denominan mucormicosis. En los últimos años han aumentado su incidencia en pacientes inmunocomprometidos, correspondiendo según diversos autores a la tercera causa de infección fúngica invasora, después de la aspergilosis y candidiasis ${ }^{1-3}$.

A ello, se agrega la dificultad para establecer un diagnóstico precoz, esencial para el tratamiento oportuno y pronóstico, debido a su rápida evolución, llegando a ser fatal en muchos casos ${ }^{1}$.

El examen histológico aproxima al diagnóstico siendo definitivo el cultivo para la identificación del género. En la actualidad la especie que se aísla con mayor frecuencia es Rhizopus oryzae ${ }^{4}$.

En Chile, las mucormicosis han sido infrecuentemente descritas en niños, existiendo publicaciones principalmente desde 1985 a la fecha y mayoritariamente en adultos con factores predisponentes ${ }^{5}$.

Presentamos un caso, en Valdivia, Chile, de mucormicosis cutánea en un niño leucémico.

\section{Caso clínico}

Preescolar con 4 años de edad, sexo masculino, residente en Valdivia. Ingresó con historia de dos semanas de evolución caracterizada por decaimiento, fatigabilidad, palidez, anorexia, fiebre no cuantificada y epistaxis autolimitada. Se le diagnosticó una leucemia linfoblástica aguda estirpe B común, de alto riesgo, por ser portador de t-9;22.

Inició quimioterapia intensiva según protocolo del
Programa Infantil Nacional de Drogas Antineoplásicas (PINDA) 2002, que incluye en la primera fase, prednisona $60 \mathrm{mg} / \mathrm{m}^{2} /$ día, vincristina $1,5 \mathrm{mg} / \mathrm{m}^{2} /$ dosis, daunorrubicina $30 \mathrm{mg} / \mathrm{m}^{2} /$ dosis, L-asparaginasa $5.000 \mathrm{U} / \mathrm{m}^{2} /$ dosis más metotrexato intratecal $12 \mathrm{mg} /$ dosis. Al día siguiente del inicio de la quimioterapia presentó una neutropenia febril con valores bajos de proteína $\mathrm{C}$ reactiva $(2,9 \mathrm{mg} / \mathrm{dl})$, recibió tratamiento antimicrobiano intravenoso empírico con cloxacilina $200 \mathrm{mg} / \mathrm{kg} /$ día y amikacina $15 \mathrm{mg} / \mathrm{kg} /$ día Los hemocultivos fueron negativos.

Evolucionó afebril, pero con neutropenia profunda. A los 12 días reapareció la fiebre, por lo que se indicó ceftriaxona $100 \mathrm{mg} / \mathrm{kg} /$ día, vancomicina $40 \mathrm{mg} / \mathrm{kg} / \mathrm{día}$ y amikacina $15 \mathrm{mg} / \mathrm{kg} /$ día, asociado a fluconazol $5 \mathrm{mg} /$ $\mathrm{kg} / \mathrm{día}^{6}$. Se confirmó una bacteriemia por Streptococcus pneumoniae, susceptible a penicilina y ceftriaxona, y una colonización faríngea por Klebsiella oxytoca.

Cuatro días después, estando afebril, pero con neutropenia profunda y prolongada, presentó una pequeña lesión micropapulo-vesicular pruriginosa en el codo derecho, interpretada inicialmente como prúrigo insectario, por lo que se indicó clorfenamina oral $0,3 \mathrm{mg} / \mathrm{kg} / \mathrm{dí}$. En los siguientes tres días progresó rápidamente a una lesión dolorosa, redondeada, ulcero-necrótica, con rodete inflamatorio violáceo en los bordes y fondo hemorrágico, de $6 \times 3 \mathrm{~cm}$ (Figura 1).

El estudio de laboratorio reveló $\mathrm{Hb} 8,9 \mathrm{gr} / \mathrm{dl}$, leucocitos 390 céls $/ \mathrm{mm}^{3}$, RAN 40 céls $/ \mathrm{mm}^{3}$, plaquetas 20.000 céls/ $\mathrm{mm}^{3}$, TTPA $21,3 \mathrm{seg}$, tiempo de protrombina $12,3 \mathrm{seg}$ (112\%), fibrinógeno $191 \mathrm{mg} / \mathrm{dl}$, dímero D 8,45 ug/dl, función hepática, renal, electrolitos y examen de orina normales.

\author{
Universidad Austral de Chile. \\ Valdivia. \\ Facultad de Medicina \\ Instituto de Pediatría (MTF, PMD). \\ Instituto de Microbiología Clínica \\ (LZC). \\ Recibido: 19 de octubre de 2010 \\ Aceptado: 18 de octubre de 2011 \\ Correspondencia a: \\ Marlis Täger Frey \\ mtager@uach.cl
}




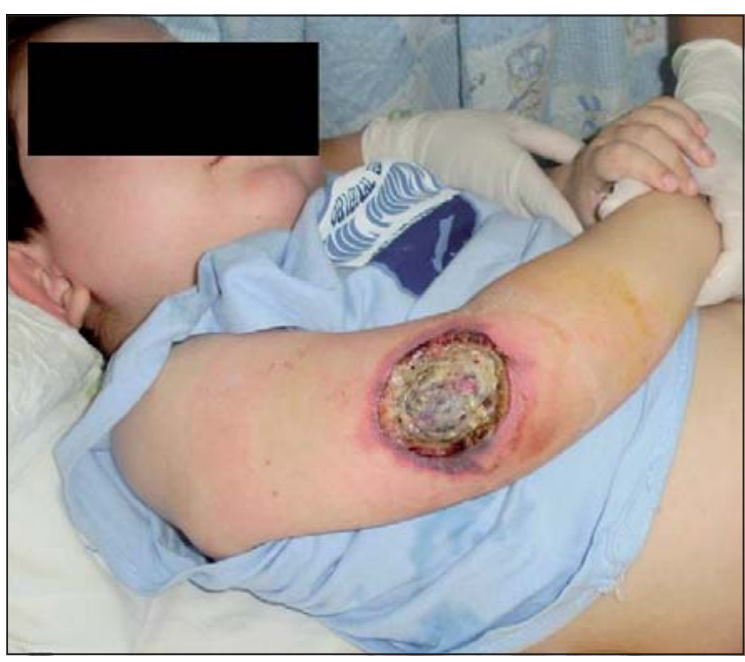

Figura 1. Mucormicosis cutánea. Lesión ulcero-necrótica del brazo, de bordes violáceos, de $6 \times 3 \mathrm{~cm}$, en un paciente con leucemia linfoblástica aguda.

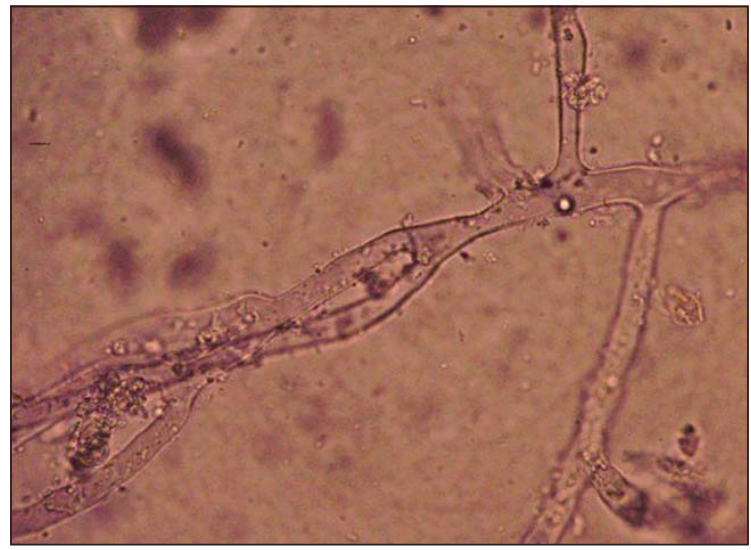

Figura 2. Hifas anchas no septadas, con ramificación en ángulo recto. Observación con $\mathrm{KOH}$-tinta, 400x.

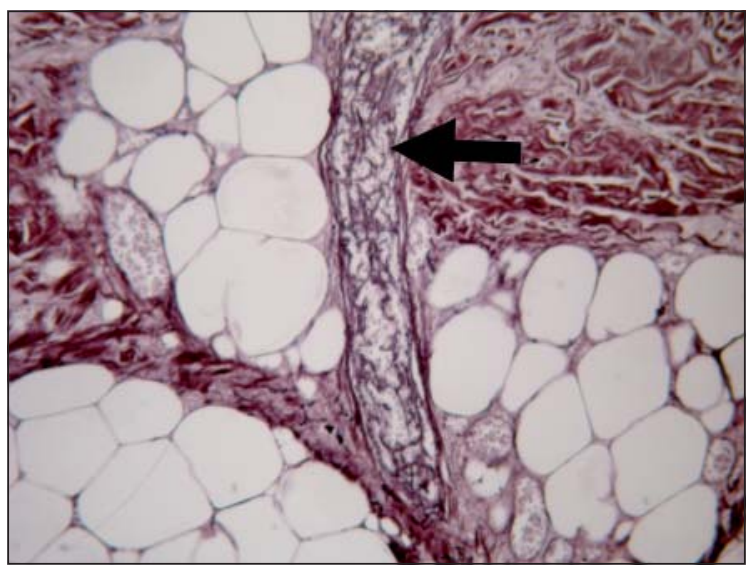

Figura 4. Vaso sanguíneo dilatado con hifas en su interior, rodeado de tejido adiposo, en dermis profundo. Tinción Gomori-Grocott.
Se planteó como diagnóstico un ectima gangrenoso y se cambió tratamiento antimicrobiano por clindamicina 40 $\mathrm{mg} / \mathrm{kg} /$ día más ceftazidima $150 \mathrm{mg} / \mathrm{kg} /$ día, manteniendo amikacina y fluconazol. Paralelamente, se realizó un aseo quirúrgico y estudio microbiológico e histológico. El cultivo corriente fue negativo. El cultivo de hongos demostró crecimiento de Rhizopus microsporus var. oligosporus (Figura 2). Un nuevo cultivo confirmó el aislado y el examen microscópico directo de la muestra con tinción de $\mathrm{KOH}$ y la histología documentaron la presencia de hifas no septadas (Figuras 3, 4 y 5).

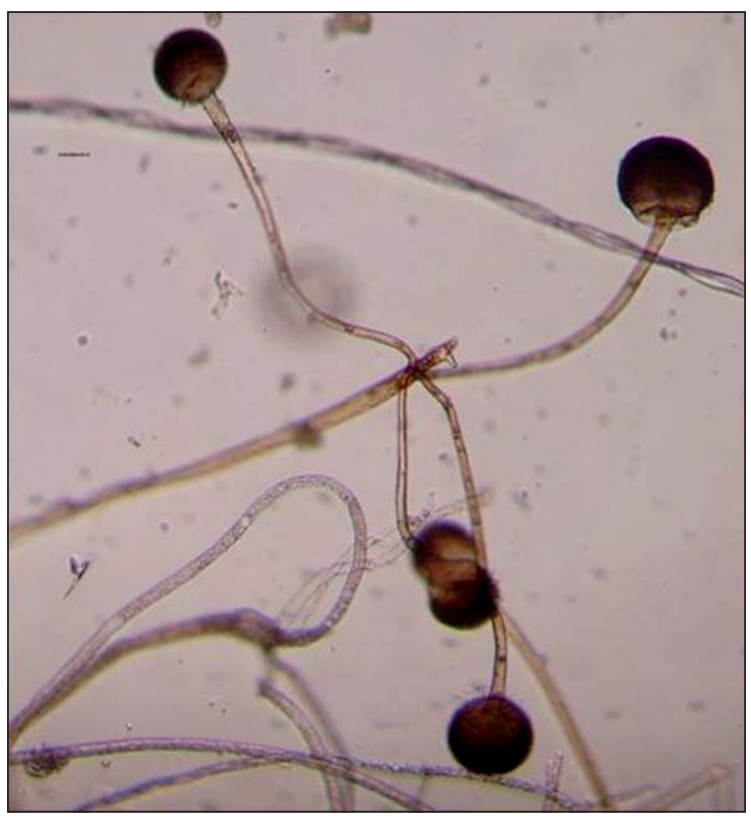

Figura 3. Rhizopus microsporus var oligosporus, con esporangio y esporangiosporas en su extremo distal, 100x.

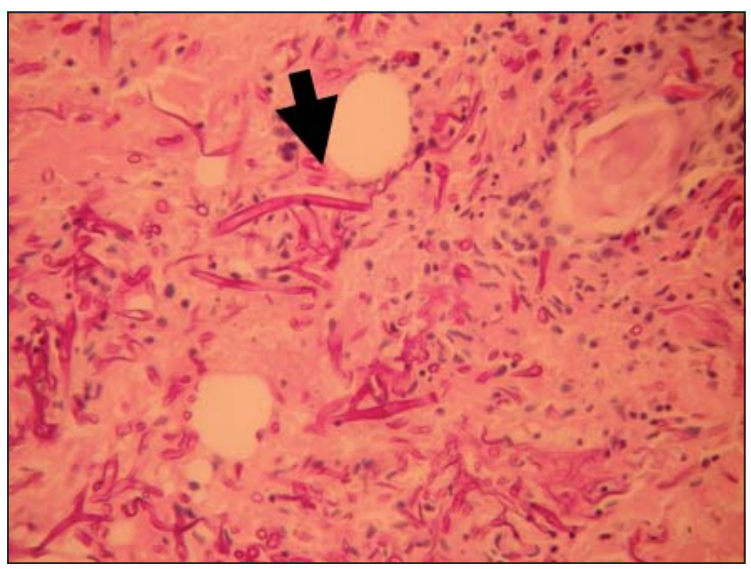

Figura 5. Hifas no septadas en color fucsia, rodeadas por tejido conectivo y restos de tejido adiposo necrótico del celular subcutáneo. Tinción PAS. 
Se cambió fluconazol por anfotericina B deoxicolato intravenosa $1,5 \mathrm{mg} / \mathrm{kg} / \mathrm{dí}$, asociado las primeras dos semanas a caspofungina $50 \mathrm{mg} / \mathrm{m}^{2} /$ día, hasta haber descartado compromiso sistémico de la infección, y suspensión transitoria de quimioterapia.

El paciente evolucionó estable, afebril, con neutropenia persistente $\mathrm{y}$, pese a repetidos aseos quirúrgicos, la escara necrótica se profundizó hasta la fascia muscular, requiriendo un desbridamiento quirúrgico hasta el subperiostio. En el último aseo, a los 22 días de tratamiento, no se encontraron elementos fúngicos en el borde de la lesión. El estudio de extensión con cintigrama óseo Tc99, TAC de cavidades paranasales y cerebral descartó compromiso óseo, sinusal y cerebral. Completó 40 días de terapia antifúngica con anfotericina $\mathrm{B}$, con evolución satisfactoria. Posteriormente se realizó un injerto dermoepidérmico (Figura 6).

\section{Discusión}

En los últimos años, la mucormicosis emerge como una importante causa de infección fúngica en pacientes con neoplasias hematológicas, especialmente en la fase de neutropenia que sigue a la quimioterapia intensiva, como en nuestro caso, a un trasplante de precursores hematopoyéticos (TPH) o de órgano sólido, representando la tercera causa de infección fúngica invasora después de Aspergillus spp. y Candida spp; comparte en algunas casuísticas esa misma posición con Fusarium $\mathrm{spp}^{1}$. Su importancia radica en la evolución fulminante de la infección diseminada con mortalidad global que alcanza a $40 \%$, pudiendo llegar hasta $95 \% \%^{7,8}$. Se la ha considerado de adquisición comunitaria, pero se está convirtiendo en una infección de adquisición nosocomial, facilitada por las terapias antifúngicas sin cobertura para mucorales, y el uso de inmunosupresores, además del aumento de la población de pacientes inmunocomprometidos ${ }^{1-3}$.

Otras poblaciones de riesgo para esta infección la constituyen pacientes con diabetes mellitus, después de cirugías de quemaduras y traumas y aquellos que reciben tratamiento con glucocorticoides o deferoxamina. En niños, se suman otras condiciones, como recién nacidos de bajo peso, cetoacidosis diabética no controlada y aciduria metabólica congénita ${ }^{9-11}$.

Dentro del orden Mucorales, los agentes más frecuentemente aislados en infección humana son especies de Rhizopus, Absidia, Mucor, Rhizomucor y Apophysomyces, predominando Rhizopus y esporádicamente Absidia ${ }^{9,10}$.

Por otra parte, ha aumentado la prevalencia de infecciones cutáneas con respecto a la presentación craneofacial y pulmonar; cambio que tiene que ver con la emergencia de Rhizopus microsporus var rhizopodiformis, como patógeno $^{11}$.

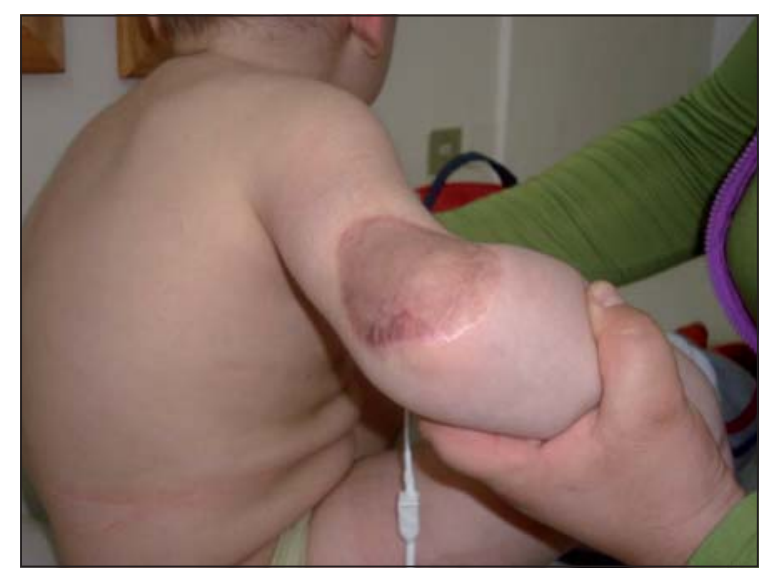

El diagnóstico precoz de estas micosis es esencial, ya que evolucionan con rapidez por su capacidad angioinvasora y el pronóstico puede ser fatal ${ }^{12}$.

Torres-Narbona, en España ${ }^{13}$, muestra que las formas cutáneas representaron el $42 \%$ de las mucormicosis. Por otra parte, en una revisión en E.U.A en niños bajo los 18 años, con infección demostrada por histología y/o cultivo, se identificaron 157 casos, donde las formas cutáneas fueron las más frecuentes, representando el $27 \%$, seguidas de las gastrointestinales $(21 \%)$, rinocerebrales $(18 \%)$ y pulmonares (16\%). Las condiciones de riesgo predominantes fueron neutropenia, prematurez y diabetes mellitus, y los agentes más importantes Rhizopus y Mucor ${ }^{14}$. En una revisión de 929 casos, reportados en la literatura científica inglesa, entre los años 1885 y 2004, Roden y col. encontraron que las formas cutáneas correspondieron al tercer lugar, después de las sinusales y pulmonares. En esta revisión, 44\% de las formas cutáneas se complicaron por profundización local o por diseminación ${ }^{11}$.

Los Mucorales son microorganismos ubicuos en la naturaleza, con una amplia distribución geográfica, encontrándose en vegetales en descomposición, semillas y frutas, pan añejo, suelo y excretas de animales e incluso en el medio hospitalario. Tienen baja virulencia, y por lo tanto, fundamentalmente ocasionan infecciones oportunistas en hospederos inmunocomprometidos. Son hongos aeróbicos cuyas colonias crecen en agar Sabouraud a temperaturas entre 25 y $55^{\circ} \mathrm{C}$ en 2 a 5 días. Las colonias son visibles a ojo desnudo y tienen aspecto algodonoso, con puntos negros tenues. La observación directa de muestras clínicas con $\mathrm{KOH}$ al $10 \%$ o con blanco de calcoflúor permite apreciar elementos filamentosos irregulares, con hifas largas, anchas y no septadas, a menudo ramificadas en ángulo recto ${ }^{1,7}$.

El modo de transmisión habitual de la enfermedad es a través de inhalación de esporas desde el ambiente, o vía percutánea con implantación traumática de esporas en zonas de disrupción cutánea, como sitios de inserción de
Figura 6. Mucormicosis cutánea tratada y curada, sometida a injerto dermo-epidérmico. 
catéteres, quemaduras, inyección de drogas o tatuajes y picaduras de insecto, ya que los agentes de mucormicosis son incapaces de penetrar la barrera cutánea intacta ${ }^{12}$. En nuestro caso, se planteó como probable puerta de entrada cutánea, una picadura de insecto, por presentar el paciente prurito y signos de grataje localizados.

En la patogenia de la enfermedad son críticas las alteraciones de los fagocitos polimorfonucleares y monucleares. En el individuo inmunocompetente los macrófagos previenen el inicio de la infección fúngica por fagocitosis y muerte oxidativa de las esporas. Una vez que la infección se establece, los neutrófilos juegan un importante rol, pues son capaces de mediar la muerte fúngica usando su sistema citotóxico oxidativo, ya que debido al gran tamaño de las hifas es imposible que sean fagocitadas por las células inflamatorias. Por ello, los hospederos inmunocomprometidos con deficiencia de granulocitos, tienen mayor riesgo de desarrollar esta infección, como en nuestro paciente ${ }^{7,12}$.

La mucormicosis se caracteriza porque las hifas tienen una gran capacidad para invadir los vasos sanguíneos, con rápida producción de trombosis y necrosis tisular. La instalación de síntomas es habitualmente aguda, siendo inusual el desarrollo de una infección silente.

De acuerdo a la presentación clínica y el compromiso anatómico específico, se conocen seis categorías clínicas: rino-orbitaria-cerebral: que comienza con la inhalación de esporas hacia los senos paranasales y puede invadir cerebro; pulmonar: que representa una forma rápidamente progresiva de neumonía con infartos y hemorragias por inhalación de esporas hacia los bronquios y alvéolos; cutánea: por la inoculación de esporas dentro de la dermis, pudiendo profundizarse localmente; gastrointestinal, que resulta de la ingestión de esporas con manifestaciones como masa abdominal, abdomen agudo, enterocolitis necrosante y peritonitis secundaria; diseminada: cuando se generaliza a otros órganos; y miscelánea ${ }^{7,9,12}$.

La mucormicosis cutánea se clasifica en dos formas: primaria, con una puerta de entrada exclusivamente cutánea, y secundaria, debido a localizaciones metastásicas de una mucormicosis rinocerebral, pulmonar o gastrointestinal ${ }^{15}$.

A su vez, la mucormicosis cutánea primaria puede ser superficial y subcutánea o gangrenosa. La primera afecta principalmente a sujetos inmunocompetentes en quienes se desarrolla una lesión necrótica de lenta evolución, relativamente circunscrita, mientras que la segunda afecta generalmente a pacientes inmunocomprometidos y/o diabéticos, quienes desarrollan lesiones necróticas extensas, de rápida evolución, penetrando desde el tejido cutáneo y subcutáneo a la grasa, músculo, fascia y eventualmente al hueso, pudiendo evolucionar a infección diseminada, cuando se extiende a otros sitios no contiguos. La invasión vascular secundaria podría originar una infección hematógena diseminada hacia órganos profundos, o generar una fascitis necrosante, ambas con elevada mortalidad ${ }^{7}$. Nuestro paciente corresponde a la forma subcutánea o gangrenosa, por presentar una lesión indurada y dolorosa, de bordes violáceos, que progresó rápidamente con necrosis tisular y profundización hasta el periostio (Figura 1).

En relación al manejo de la mucormicosis, cuatro factores son críticos para erradicar la infección:

- Rapidez en el diagnóstico, que requiere un alto índice de sospecha clínica y realización de una biopsia quirúrgica con implementación de un diagnóstico rápido (intra-operatorio) mediante análisis del tejido en fresco con examen microscópico directo con $\mathrm{KOH}$-tinta y/o tinción de blanco-calcoflúor, además del estudio microscópico de tejidos fijados mediante tinciones de hematoxilina-eosina, PAS, o Gomori-Grocott (Figuras 2, 4 y 5). Estos criterios permiten identificar una infección por hongos de la familia Mucoraceae y diferenciar de hongos filamentosos tabicados como Aspergillus sp. y Fusarium sp.

- Corrección, en lo posible, de los factores predisponentes como hiperglicemia en diabéticos y neutropenia en pacientes con cáncer, y reducir o suspender transitoriamente los fármacos inmunosupresores, particularmente corticoesteroides $^{15,16}$.

- Desbridamiento quirúrgico exhaustivo y urgente de los tejidos infectados, ya que la mucormicosis es una infección rápidamente progresiva y la terapia antifúngica sola es incapaz de controlar la infección, por la trombosis vascular y extensa necrosis tisular que se produce, que impide la buena llegada del antifúngico. La mucormicosis cutánea tiene un pronóstico favorable, con mortalidad menor al 10\%, cuando se realiza diagnóstico y desbridamiento quirúrgico precoz de la lesión, requiriendo como en nuestro paciente (Figura 6), de cirugía reconstructiva pasada la fase aguda ${ }^{12,14}$.

- Tratamiento antifúngico, siendo de primera línea, para la mucormicosis invasora, anfotericina B deoxicolato, en dosis de 1-1,5 mg/kg/día. Las formulaciones lipídicas de anfotericina son significativamente menos nefrotóxicas y pueden ser administradas a mayores dosis y por períodos más prolongados de tiempo ${ }^{16}$. Una revisión retrospectiva de 120 casos de mucormicosis en pacientes con neoplasias hematológicas mostró que el tratamiento con anfotericina liposomal fue asociado a una tasa de sobrevida de $67 \%$ comparado con $39 \%$ de los tratados con anfotericina deoxicolato ${ }^{17}$.

Las equinocandinas, antifúngicos de baja toxicidad, no inhiben a los mucorales ${ }^{16}$, aunque se ha visto efectos sinérgicos en estudios in vitro con caspofungina y posaconazol, y en múridos entre anfotericina $\mathrm{B}$ liposomal y equinocandinas. Caspofungina ha demostrado inhibir la síntesis de 
$\beta$-glucanos de la pared de Rhizopus oryzae, al impedir la acción de la enzima $1,3-\beta$-D-glucano sintetasa ${ }^{18}$.

Publicaciones recientes, basadas en estudios preclínicos en modelos murinos, como también en terapia en pacientes inmunocomprometidos de polienos con caspofungina, han mostrado mejor evolución en mucormicosis rino-orbital y rino-orbito-cerebral en relación a monoterapia con anfotericina. Siguiendo estos criterios, usamos anfotericina B deoxilato asociada inicialmente a caspofungina.

El rol de la terapia combinada como tratamiento de primera línea de mucormicosis, ha sido objeto de reciente revisión, concluyéndose que faltan estudios clínicos prospectivos y randomizados para determinar si la combinación de polienos con equinocandinas es superior a la monoterapia con anfotericina ${ }^{19-21}$.

En ausencia de datos comparativos, la duración de la terapia debería ser individualizada para cada paciente, y continuada hasta la resolución clínica de la infección, estabilización y/o normalización de los signos radiográficos y la recuperación de la inmunosupresión subyacente $e^{12,18,19,21}$.

Para pacientes que están recibiendo inmunosupresores, se debe continuar con profilaxis antifúngica secundaria por todo el período, siendo posaconazol una alternativa a los polienos. En pacientes con inmunosupresión intermitente por ciclos de quimioterapia, la profilaxis secundaria debería instalarse durante los períodos de neutropenia que siguen a ésta ${ }^{6}$.

La bibliografía chilena sobre mucormicosis en niños es escasa, existiendo publicaciones mayoritariamente en adultos con factores predisponentes.

En los últimos 25 años, encontramos reportados: en 1985, una paciente con leucemia aguda monoblástica publicada por Conte y cols ${ }^{5}$, y dos casos de zigomicosis orbitaria y cerebral en adultos, comunicados por Cox y cols $^{22}$. En 1987, un caso de mucormicosis orbitaria en un adulto diabético descrito por Villarroel ${ }^{23}$, y una publicación de Thompson y cols., que revisan indicaciones para el diagnóstico y tratamiento de micosis oportunistas. Para esa fecha, se habrían comunicado alrededor de 30 casos, preferentemente en pacientes diabéticos ${ }^{24}$.

En 1988, Oddó y Acuña, en un estudio retrospectivo sobre la base de 5.612 autopsias, realizadas entre 1960 y 1986, encontraron, de acuerdo a criterios morfológicos, 175 infecciones oportunistas predominantemente micóticas, representando las zigomicosis 5,7\% de los casos ${ }^{25}$. Godoy, en 1999, reporta una mucormicosis rino-orbitaria en un adulto con leucemia crónica ${ }^{26}$.

García y cols, el año 2001 comunican casos de mucormicosis rino-orbital en dos adultos inmunocomprometidos, identificando Rhizopus $\mathrm{sp}^{27}$. Bertin, el 2003 en Valdivia, informa una mucormicosis rinosinusal en un adulto diabético ${ }^{28}$. El 2004, Aguad y cols., comunican un caso de zigomicosis rinocerebral en una mujer con infec- ción por $\mathrm{VIH}^{29}$, Chávez y cols, informa una mucormicosis rinocerebral en un niño diabético ${ }^{30}$, Spalloni y cols y Wicki y cols describen dos casos de mucormicosis rinocerebral en pacientes pediátricos con leucemia ${ }^{31,32}$.

Iñiguez y cols, el 2006, describen una mucormicosis rino-orbitaria en una paciente de 13 años, con leucemia linfática aguda y neutropenia secundaria a quimioterapia ${ }^{33}$.

Fernández, el 2005, revisa los hallazgos clínicos, patológicos, y tratamiento en 12 pacientes con mucormicosis en el Hospital Clínico de la Universidad de Chile, en Santiago, entre 1990 y 2004. El diagnóstico se realizó por biopsia en 10 pacientes y por estudio directo en dos. Los factores predisponentes fueron, diabetes mellitus, SIDA y enfermedades hemato-oncológicas. La histología demostró necrosis e infarto con invasión de vasos sanguíneos y trombosis micótica. Todos los pacientes recibieron tratamiento con anfotericina B y 6 fueron intervenidos quirúrgicamente. Del total, 33\% falleció ${ }^{34}$.

Rahal y cols., el año 2008 revisan la casuística de 12 años de mucormicosis rino-orbito cerebral entre 1993 y 2004, del Hospital Barros Luco Trudeau, en Santiago, analizando un total de 16 pacientes; sólo uno inmunocompetente. Llaman la atención sobre la baja frecuencia de presentación de estos cuadros y su alta mortalidad. El síntoma más frecuente fue dolor facial y el hallazgo más observado al examen físico fue la presencia de una escara negra o grisácea en la mucosa nasal. El examen micológico directo permitió visualizar el hongo en 11 casos y el cultivo resultó positivo en 10 pacientes. El $62,5 \%$ de los pacientes sobrevivió a la micosis. Rhizopus oryzae fue el agente identificado en el $90 \%$ de los $\operatorname{casos}^{35}$.

Villanueva y cols, presentan el año 2009 una mucormicosis intestinal en un neonato de término ${ }^{36}$. Las últimas publicaciones, el 2011, son de Tapia y cols, que informan dos casos, en Temuco, de zigomicosis cutánea tratados con anfotericina B deoxicolato, falleciendo uno de los pacientes ${ }^{37}$, y de Cruz y Piontelli, en Valparaíso, que comunican 51 casos de infección fúngica invasora en pacientes mayoritariamente inmunocomprometidos, entre el 2004 y 2009, donde sólo uno correspondió a mucormicosis, aislándose Rhizopus oryzae ${ }^{38}$.

Al revisar la literatura científica chilena, la mayoría de los casos de mucormicosis fueron diagnosticados por histopatología, siendo algunos hallazgos de laboratorio, desconociéndose en muchos la especie del hongo causal. En nuestro caso, la rapidez en el diagnóstico micológico con identificación del hongo, como la adecuada terapia antifúngica y el aseo quirúrgico, contribuyeron a una evolución favorable de la micosis.

Agradecimientos. A Eduardo Alvarez, del Laboratorio de Micología de la Universidad Rovira y Virgili, Cataluña, España, por la confirmación de la especie del hongo aislado. 


\section{Caso Clínico}

\section{Resumen}

La mucormicosis es una enfermedad emergente grave, producida por hongos saprófitos del orden Mucorales, que afecta fundamentalmente a pacientes inmunocomprometidos. La forma cutánea se origina por inoculación de esporas dentro de la dermis con el subsecuente desarrollo de una lesión tipo ectima, generalmente única y de evolución rápidamente progresiva, por las características angioinvasoras del hongo, que determina amplias zonas de infartos y necrosis en los tejidos. Presentamos el caso de un paciente con cuatro años de edad, con diagnóstico de leucemia linfoblástica aguda, en quimioterapia de inducción, que cursó con neutropenia profunda y prolongada, presentando una lesión cutánea en el codo compatible con ectima gangrenoso. Recibió tratamiento antimicrobiano y antifúngico, asociado a un aseo quirúrgico. El cultivo para hongos demostró crecimiento de Rhizopus microsporus var oligosporus, y la histología concluyó presencia de hifas no septadas. El estudio de extensión descartó compromiso óseo, sinusal y cerebral. Completó 40 días de terapia antifúngica con anfotericina B deoxicolato, evolucionando satisfactoriamente. Posteriormente requirió injerto dermo-epidérmico. Si bien esta patología es infrecuente, debemos sospecharla en pacientes con neoplasias hematológicas, para establecer un diagnóstico etiológico oportuno, ya que el tratamiento contempla el desbridamiento quirúrgico precoz asociado a antifúngicos sistémicos, siendo de elección anfotericina B.

\section{Referencias}

1.- Leventakos K, Russell E L, Kontoyiannis D P. Fungal infections in leukemia patients: How do we prevent and treat them? Clin Infect Dis 2010; 50: 405-15.

2.- Torres-Narbona M, Muñoz J, Guinea P, Bouza E. Zigomicetos y zigomicosis en la era de las nuevas terapias antifúngicas. Rev Esp Quimioterap 2007; 20: 375-86.

3.- Kontoyiannis D P, Marr K A, Park B J, Alexander B D, Anaissie E J, Walsh T J, et al. Prospective surveillance for invasive fungal infections in hematopoietic stem cell transplant recipients 2001-2006: Overview of the Transplant-Associated Infection Surveillance Network (TRANSNET) Database. Clin Infect Dis 2010; 50: 1091-100.

4.- Lass-Flörl C. Zygomycosis: Conventional laboratory diagnosis. Clin Microbiol Infect 2009; 15 (Suppl. 5): 60-5.

5.- Conte G, Thompson L, Rohmann I, Oddo D, Rojas P, Riquelme R. Mucormicosis en un paciente con leucemia aguda monoblástica. Rev Chil Infectol 1985; 2: 147-51.

6.- Santolaya M E, Alvarez A, Avilés C, Becker A, Cofré J, Palla E, et al. Tratamiento selectivo de los episodios de neutropenia febril en niños con cáncer. Comité de Infectología, Programa Infantil Nacional de Drogas Antineoplásicas (PINDA). Rev Chil Infectol 2004; 21: 213-22.

7.- Spalloni P, Chávez A, Avilés C, Cofré J. Mucormicosis en Pediatría. Rev Chil Infectol 2004; 21: 17-25.

8.- Bethge W, Schmalzing M, Stuhler G, Schumacher U, Kröber S, Horger M, et al. Mucormycoses in patients with hematologic malignances: an emerging fungal infection. Haematologica 2005; 90: e62-4.

9.- Ribes J, Vanover-Sams C, Baker D. Zygomycetes in human disease. Clin Microbiol Rev 2000; 13: 236-301.
10.- Kauffman C. Zygomycosis: Reemergence of an old pathogen. Clin Infect Dis 2004; 39: 588-90.

11.- Roden M, Zaoutis T, Buchanan W, Knudsen T, Sarkisova T, Schaufele R, et al. Epidemiology and outcome of zygomycosis: A review of 929 reported cases. Clin Infect Dis 2005; 41: 634-53.

12.- Spellberg B, Edwards J, Ibrahim A. Novel perspectives on mucormycosis: Pathophysiology, presentation and management. Clin Microbiol Rev 2005; 18: 556-69.

13.- Torres-Narbona M, Guinea J, Martínez-Alarcón J, Peláez T, Muñoz P, Bouza E. Zygomicosis in a general hospital during a 17 year period. Is there an increase incidence after introducing voriconazole? $16^{\text {th }}$ ECCMID, Niza 2006, abst P 1201.

14.- Zaoutis T, Roilides E, Chiou C, Buchanan W, Knudsen T, Sarkisova T, et al. Zygomycosis in children: A systematic review and analysis of reported cases. Pediatr Infect Dis J 2007; 26: 723-7.

15.- Pagano L, Offidani M, Fianchi L, Nosari A, Candoni A, Piccardi M, et al. Mucormycosis in hematologic patients. Haematologica 2004; 89: 213-4.

16.- Spellberg B, Walsh T, Kontoyiannis D, Edwards J, Ibrahim A. Recent advances in the management of mucormycosis: From bench to bedside. Clin Infect Dis 2009; 48: 1743-51.

17.- Gleissner B, Schilling A, Anagnostopolous I, Siehl I, Thiel E. Improved outcome of zygomycosis in patiens with hematological diseases. Leukemia Lymphoma 2004; 45: 1351-60.

18.- Reed C, Bryant R, Ibrahim A, Edwards J, Filler S, Goldberg R, et al. Combination polyene-caspofungin treatment of rhino-orbitalcerebral mucormycosis. Clin Infect Dis 2008; 47: 364-71.

19.- Walsh T, Kontoyiannis D. What is the role of combination therapy in management of zygomycosis? Clin Infect Dis 2008; 47: 372-4.

20.- Vásquez L, Mateos J, Sanz-Rodríguez C, Pérez E, Caballero D, San Miguel J F. Successful treatment of rinocerebral zygomycosis with a combination of caspofungin and liposomal amphotericin B. Haematologica 2005; 90: 109-10

21.- Spellberg B, Ibrahim A S. Recent advances in the treatment of mucormycosis. Curr Infect Dis Rep 2010; 12: 423-9.

22.- Cox J, Corona S, Donoso S. Mucormicosis orbitaria y cerebral en dos pacientes diabéticos. Rev Med Chile 1985; 113: 334-41.

23.- Villarroel F. Mucormicosis orbitaria (Ficomicosis, Zigomicosis). Arch Chil Oftalmol 1985; 42: 61-5.

24.- Thompson L, Oddo D, Retamal C, Ferrada L. Orientaciones clínicas y de laboratorio en las micosis oportunistas. Rev Chil Infectol 1987;4: 69-74.

25.- Oddo D, Acuña G. Infecciones oportunistas en 5.612 necropsias. Santiago, Chile, 1960-1986. Bol Of Sanit Panam 1988;22: 251-60.

26.- Godoy J. Mucormicosis rino-orbitocerebral. Rev Med Chile 1999;10: 67-70.

27.- García P, Beltrán C, Guzmán A, León P, Arredondo $\mathrm{M}$, Fonsaca X. Diagnóstico rápido de dos casos de mucormicosis con tinción de blanco de calcoflúor. Rev Chil Infectol 2001; 18: 285-90.

28.- Bertín H. Mucormicosis rinosinusal. Rev Otorrinolaringol Cir Cabeza Cuello 2003;63: 122-26.

29.- Aguad L, Quezada M, Rahal M, Vallejos M, Moreno M, Castillo J, et al. Mucormicosis rinosinusal en un paciente infectado por VIH. Rev Chil Infectol 2004; 21: 345-50.

30.- Chávez A, Rahal M. Mucormicosis rinocerebral en un niño diabético: Tratamiento conservador Rev Chil Infectol 2004; 21: 57-60.

31.- Spalloni P, Glaser P, and Verdugo P. Mucormicosis rinocerebral: Sobrevida en un 


\section{Caso Clínico}

niño con leucemia. Rev Chil Infectol 2004; 21: 53-6.

32.- Wicki A, Borel C, Villarroel M, Cofré J. Curación de mucormicosis rinocerebral en un paciente pediátrico con leucemia activa. Rev Chil Infectol 2004; 21: 48-52.

33.- Iñiguez R, Cevo J, Fonseca X. Terapéutica de apoyo en la mucormicosis. Rev Otorrinolaringol Cir Cabeza Cuello 2006; 66: 236-42.

34.- Fernández E, Dünner M, Mandiola C, Silva M.
Experiencia en mucormicosis. Hospital Clínico Universidad de Chile. Revista Hospital Clínico Universidad de Chile 2005; 16: 198-203.

35.- Rahal M, Moreno M, Villa J. Mucormicosis rino-orbitocerebral: Experiencia en doce años. Rev Otorrinolaringol Cir Cabeza Cuello 2008; 68: 27-34.

36.- Villanueva Ch M, Avilés C L. Mucormicosis intestinal: Reporte de un caso en neonato de término. Rev Chil Pediatr 2009. Libro de resúmenes XLIX Congreso Chileno de Pediatría.

37.- Tapia O, Chahín C, Concha C. Mucormicosis cutánea primaria: a propósito de dos casos. Revisión de la literatura. Rev Chil Infectol 2011; 28: 269-73.

38.- Cruz R, Piontelli E. Enfermedad fúngica invasora en pacientes de cinco hospitales de la Región de Valparaíso, Chile: 2004 a 2009. Rev Chil Infectol 2011; 28: 123-9. 